\title{
La enseñanza estratégica para abordar la composición de textos narrativos
}

\author{
Strategic teaching to address the composition of narrative texts
}

Recibido febrero 2019

Arbitrado marzo 2019

Publicado mayo 2019
Norka Pérez

norkaperezeduca@gmail.com

Código ORCID: 0000-0002-8897-4066

Escuela Básica Dr. Ramón Alfonso Blanco, Venezuela

\section{Resumen}

El presente estudio se planteó formular un grupo de estrategias instruccionales basadas en la enseñanza estratégica para abordar la composición de textos narrativos. La metodología utilizada se fundamentó en el enfoque cualitativo, con un diseño de investigación de campo con carácter descriptivo e interpretativo. Los participantes fueron estudiantes de cuarto grado de Educación Primaria del Grupo Escolar Guatire del estado Miranda, Venezuela. Las técnicas de recolección de la información fueron la entrevista semiestructurada y la observación-participante. Como instrumentos se utilizaron el guion de entrevista y la guía de observación. Entre los resultados obtenidos está que los docentes en este nivel de Educación Primaria no emplean estrategias instruccionales novedosas ni creativas que orienten a los aprendices en la composición de textos escritos. Por lo que, se diseñaron estrategias instruccionales fundamentadas en la enseñanza estratégica atendiendo a los intereses y motivaciones de los estudiantes.

\footnotetext{
Abstract

The present study set out to formulate a group of instructional strategies based on strategic teaching to address the composition of narrative texts. The methodology used was based on the qualitative approach, with a descriptive and interpretive field research design. The participants were fourth grade students of Primary Education of the Guatire School Group from the state of Miranda, Venezuela. The information collection techniques were the semi-structured interview and the participant-observation. The interview script and the observation guide were used as instruments. Among the results obtained is that teachers at this level of Primary Education do not use innovative or creative instructional strategies that guide learners in the composition of written texts. Therefore, instructional strategies based on strategic teaching were designed taking into account the interests and motivations of the students.
}

\author{
Palabras clave: \\ Estrategias \\ instruccionales; \\ enseñanza \\ estratégica; \\ composición de \\ textos; textos \\ narrativos escritos
}

\author{
Keywords: \\ Instructional \\ strategies; strategic \\ teaching; \\ composition of \\ texts; written \\ narrative texts
}




\section{INTRODUCCIÓN}

$\mathrm{D}$ esde hace algún tiempo, la escuela venezolana ha sido objeto de constantes renovaciones debido a la presencia de propuestas pedagógicas abordadas por los investigadores que se dedican a indagar lo que ocurre en el aula.

Desde España, el psicólogo Monereo (2004) plantea la instrucción basada fundamentalmente en enseñar para la autonomía; entendiéndose esta última como "la facultad de tomar decisiones que permitan regular el propio aprendizaje para aproximarlo a una determinada meta" (p. 4). Cuando este autor plantea "enseñar para la autonomía", está proponiendo al docente facilitar a sus estudiantes herramientas que les permitan aprender por sí mismos, haciéndoles conscientes del conocimiento que tienen e induciéndolos a decidir cuándo y cómo usarlo para resolver tareas asignadas superando dificultades que se pudieran presentar.

Dicho de otro modo, ser autónomos aprendiendo implica reflexionar sobre cómo apropiarse del conocimiento y a tomar la decisión al emplear una u otra estrategia para aprender algún contenido.

La toma de decisiones por parte de los aprendices sería muy difícil sin el entrenamiento adecuado; es por ello que las escuelas demandan contar con docentes estratégicos, capaces de facilitar las herramientas necesarias para que ellos resuelvan sus tareas de forma independiente.

En este particular, Gaskins y Pressley (2006) señalan que:

Se considera que un docente es estratégico si está en condiciones de ajustar, de manera deliberada, sus prácticas al contexto de cada materia (objetivos, contenidos, alumnos, recursos, competencias personales, etcétera) y más específicamente, si es capaz de autoregular sus acciones frente a demandas que pudiesen producirse en su aula, de manera inesperada. Desde este planteamiento la toma de decisiones cognitivas sobre qué hacer y decir en cada circunstancia sería suficiente para resolver cualquier eventualidad. (p. 2)

Lo anterior explica la importancia de un docente estratégico en la adquisición de conocimiento por parte de los aprendices, ya que el docente deberá planificar y abordar los contenidos con estrategias sencillas y flexibles. Además debe reconocer que 
cuando se trabaja con niños hay que aprovechar su espontaneidad y permitirles realizar las tareas con ciertas exigencias que contribuyan al logro de la meta en forma divertida, eficaz y dinámica procurando su participación activa por ser los protagonistas de su propio aprendizaje.

Esas estrategias estarán dirigidas por el docente y tendrán la intención de lograr el avance de los educandos ávidos de conocimiento, siempre respetando sus procesos, su desarrollo físico, intelectual y socioemocional. Ese tipo de estrategias son denominadas por algunos autores, como estrategias instruccionales. En tal sentido, se deben organizar todas y cada una de las situaciones de enseñanza y aprendizaje, considerando los componentes que garantizarán el logro de la meta propuesta que constituye el "cómo" del proceso o la forma de conducir en sí el hecho instruccional.

Feo (2010) señala que "para el logro de las estrategias de aprendizaje de manera independiente y autorregulada, el docente debe proponer estrategias instruccionales que propicien en los estudiantes la habilidad de reflexionar sobre la forma que ellos mismos emplean para aprender" (p. 222). Por ello, estas estrategias desarrolladas dentro del aula como espacio principal para los procesos de enseñanza y aprendizaje deben crear condiciones apropiadas que respondan a las necesidades del grupo y que conduzcan a la adquisición del conocimiento y a la comprensión del cómo aprenden en forma reflexiva, divertida y amena.

Es en el aula donde el docente debe poner de manifiesto toda su creatividad y utilizar las herramientas con la intención de atraer la atención del estudiante mediante la motivación; mientras que el estudiante, por su parte, asumirá una participación activa y responsable de su propio proceso de formación. Al respecto, Lomas (2001) expresa:

... el aula ya no es solo el escenario físico del aprendizaje escolar, sino también ese escenario comunicativo donde se habla y se escucha (y donde algunos se distraen), donde se lee y se escribe, donde unos se divierten y otros se aburren, donde se hacen amigos y enemigos, donde se aprenden algunas destrezas, hábitos y conceptos a la vez que se olvidan otras muchas cosas. (p. 10)

En este aparte es necesario considerar el aporte de Vygotsky (1979), que dejó un legado teórico trascendente en el ámbito de la 
psicología y la educación y entre cuyas tesis destaca el impacto que produce el contexto en el estudiante. Este autor trata sobre procesos de interiorización los cuales se expresan claramente en el siguiente planteamiento:

Cualquier función en el desarrollo cultural del niño aparece en escena dos veces, en dos planos: primero como algo social, después como algo psicológico; primero entre la gente, como una categoría interpsíquica, después, dentro del niño, como una categoría intrapsíquica. (p. 161)

Se puede entender entonces que en la categoría interpsíquica, todo comienza con la interacción social del niño con el medio que lo rodea; es decir, en principio el núcleo familiar, luego se va ampliando con familiares cercanos y paulatinamente crece hasta llegar a la escuela y aún más allá, en la cual los adultos que lo educan inculcan en ellos la cultura y la idiosincrasia del país donde nace el niño. Lo anterior da pie a que se desarrolle la categoría intrapsíquica, permitiendo la aparición del lenguaje privado, del lenguaje interior y del pensamiento verbal.

Desde esta perspectiva, para el docente estratégico el currículo en todas sus áreas proporciona metodología y herramientas que impulsan el desarrollo de una competencia básica tan necesaria en este nivel de escolaridad como es la escritura; apoyándose en la familia como la institución de mayor importancia en el proceso psicológico del niño. Por tanto, los docentes deben dedicar esfuerzos para desarrollar dicha competencia en sus educandos.

Por su parte, el Currículo Básico Nacional (Ministerio de Educación, 1997), en el bloque correspondiente a la "Literatura: El mundo de la imaginación", hace referencia a las experiencias de aprendizaje relacionadas con el disfrute y la recreación que permite la interacción con la literatura, y promueve la creatividad y el desarrollo de la fantasía en la producción espontánea de textos imaginativos ofreciendo un espacio para la evocación y la ensoñación en la formación de escritores autónomos y productores de textos creativos. Además, este bloque cumple con la importante función de permitir al estudiante su enriquecimiento personal, el conocimiento del acervo cultural de su contexto social, la reafirmación de su identidad y favorece el pensamiento divergente. 
La escritura forma parte de la cotidianidad y su dominio es un factor decisivo para el desenvolvimiento académico y social. Díaz (2005) explica que la escritura, al igual que la lectura, requiere de constancia y dedicación; por lo tanto, leer y escribir son dos actividades complementarias y necesarias para consolidar el proceso educativo del individuo, así como la comprensión y la composición de textos escritos. Es aquí donde el docente juega un papel fundamental en el desarrollo intelectual y la capacidad de redacción de los educandos.

Es importante señalar que la composición de textos es compleja y requiere de una práctica guiada y constante, por ello el docente debe emplear una metodología adecuada aplicando estrategias idóneas que ayuden a los estudiantes a apropiarse del conocimiento ya que en el nivel de Educación Primaria es donde se crean los cimientos de tan importantes competencias comunicativas.

Uno de los objetivos primordiales de la Educación Primaria es que los estudiantes dominen la lectura y la escritura, para el logro de la comprensión y composición de textos imaginativos; sin embargo, en las instituciones escolares el escenario es otro, debido a que muchos docentes no saben explicar el tipo de estrategias que usan para lograr esos objetivos.

El Currículo Básico Nacional (Ministerio de Educación, 1997) plantea: “... el propósito de la enseñanza y aprendizaje en el nivel de Educación Básica es lograr el desarrollo de la competencia comunicativa del aprendiz atendiendo a los procesos de comprensión y producción en el lenguaje oral y escrito" (p.130).

Ciertamente, mientras los docentes se ocupen de corregir los aspectos formales de la escritura en las producciones de sus estudiantes y no los orientan para que compongan textos narrativos donde se evalúe la creatividad, la coherencia, la expresión de sus pensamientos y sus sentimientos, será imposible desarrollar competencias y habilidades inherentes a la composición escrita para que el estudiante prosiga sus estudios de forma exitosa.

Esta problemática quedó evidenciada en la presente investigación, a través de las observaciones realizadas a los docentes de la institución, en la que se pudo detectar clases tradicionales y monótonas donde todo lo hace el docente, también se observó que no se sienten motivados para buscar alternativas en la enseñanza y aprendizaje de la lengua escrita; adicional a esto, algunos maestros presentan dificultad cuando deben explicar 
sus acciones dentro del aula aun cuando se les concede tiempo para la planificación.

Por consiguiente, se hace necesario proporcionar estrategias que permitan al docente analizar la realidad mediante la instrucción. Por este motivo la investigación se planteó como objetivo general proponer estrategias instruccionales basadas en la enseñanza estratégica para abordar la composición de textos narrativos en estudiantes de cuarto grado de Educación Primaria en el Grupo Escolar Guatire, estado Miranda, y como objetivos específicos:

1. Determinar las estrategias que utilizan los docentes para orientar la composición de textos narrativos en estudiantes de cuarto grado de Educación Primaria en el Grupo Escolar Guatire, estado Miranda.

2. Diseñar estrategias instruccionales basadas en la enseñanza estratégica que permitan promover la composición de textos narrativos en estudiantes de cuarto grado de Educación Primaria en el Grupo Escolar Guatire, estado Miranda.

\section{Estrategias instruccionales}

La estrategia instruccional es "la organización del ambiente y de los eventos de instrucción a fin de lograr los objetivos propuestos" (Gagné y Briggs, 1978, p. 97). Para Díaz Barriga y Hernández (2010), las estrategias instruccionales se definen como "procedimientos (conjuntos de operaciones o habilidades), que un docente emplea en forma consciente, controlada e intencional como instrumentos flexibles para enseñar significativamente y solucionar problemas" (p. 269).

Por su parte, Alfonzo (2003) considera que:

La estrategia instruccional es la organización secuencial, que realiza el docente, del contenido a aprender, la selección de los medios instruccionales idóneos para presentar ese contenido y sobre todo la organización de los estudiantes para ese propósito. Tiene dos aspectos fundamentales que son la audiencia y el contenido, teniendo como propósito promover el aprendizaje. (p. 2)

Esta definición propone organizar la secuencia del contenido y la audiencia para de esa forma obtener un mejor resultado en el 
aprendizaje de los estudiantes. Estas estrategias son clasificadas por Alfonzo (2003), como estrategias de inicio, desarrollo y cierre.

Como se puede observar en las definiciones anteriores los adelantos son notorios debido a que en cada investigación realizada se van descubriendo factores determinantes para lograr con más precisión el objetivo principal, que es darles herramientas a los estudiantes para alcanzar la independencia en la construcción de su aprendizaje.

Para Díaz Barriga y Hernández (2010), las estrategias pueden ser clasificadas en:

Estrategias preinstruccionales (al inicio): Tienen como finalidad que el estudiante sea capaz de plantearse objetivos y metas, que le permitan al profesor saber si el estudiante tiene idea de lo que la asignatura contempla y la finalidad de su instrucción.

Estrategias coinstruccionales (durante): Son aquellas que apoyan los contenidos curriculares durante el proceso de enseñanza.

Estrategias posinstruccionales (al término): Son aquellas que se presentan después del contenido que se ha de aprender.

La estrategia constituye entonces una de las piezas fundamentales de los procesos de enseñanza y aprendizaje, puesto que de ella depende la orientación y operatividad del proceso e implica una interrelación con los demás elementos del diseño instruccional, como lo son: los objetivos, los contenidos, las conductas de entrada de los alumnos, los medios instruccionales y la evaluación.

Para efectos de esta investigación se explican cada uno de los momentos y eventos instruccionales, según Alfonzo (2003). Estos momentos y eventos fueron pilares guías para las estrategias instruccionales que se presentan, considerando que estarán dirigidas a una audiencia del nivel de Educación Primaria y al tipo de contenidos u objetivos del currículo. Al respecto, este autor plantea lo siguiente:

Momento del inicio: se prepara al estudiante para la instrucción, se activa la atención y se promueve la motivación y el interés. Los cuatro eventos instruccionales señalados para el momento de inicio son los siguientes: activar la atención, establecer el 
propósito, incrementar el interés y la motivación y presentar la visión preliminar de la lección.

Momento del desarrollo: en este momento instruccional se presenta y procesa la nueva información. Se busca recordar conocimientos previos, profesar la nueva información, focalizar la atención, usar estrategias de aprendizaje, practicar lo aprendido.

Momento de cierre: el momento instruccional de cierre tiene el propósito de revisar el aprendizaje logrado para utilizarlo en diferentes contextos y abrir la posibilidad de adquirir o construir nuevos aprendizajes y de establecer enlaces con otros contenidos. Para ello se debe revisar y resumir la lección, transferir el aprendizaje, remotivar y cerrar, proponer enlaces.

Todo esto describe los eventos instruccionales que forman parte de la estrategia instruccional propuesta y constituye una guía sistemática para la planificación de acciones educativas, no obstante, en la práctica pedagógica algunos de ellos se omiten o se mezclan.

\section{La escritura}

Cassany (1990) afirma que escribir es una forma de utilizar el lenguaje, realizar acciones para conseguir objetivos, aprender a utilizar las palabras para que signifiquen lo que uno pretende que signifiquen en cada contexto. Con base en esto, la escritura es la manera como se pueden expresar las ideas, para así fomentarlas y dar a conocer los pensamientos, ya sean emociones, experiencias, conocimientos, ideales, etc.

Cuando el niño se enfrenta al acto de escribir para expresar pensamientos y sentimientos, lo hace desde su contexto social y de esa forma genera ideas que le permiten ampliar su visión del entorno, por tanto reflexiona sobre lo que va escribir.

Para Caldera (2003) "la habilidad de escribir no es innata sino adquirida." Entonces, componer implica poner en funcionamiento diversos conocimientos: a) social, conocimiento sobre las relaciones entre interlocutores y su forma de reglamentarlas (situación comunicativa); b) conceptual, el tema el contexto, y c) lingüístico, repertorio de formas lingüística específicas del tipo de texto. 
Cassany et al., (2000) plantean que "partiendo de las teorías cognitivas, lingüísticas, psicolingüísticas, sociolingüística y constructivista establecen una clasificación extensa de estrategias para la expresión escrita que tienen finalidades didácticas" (p. 268).

Estas estrategias se presentan en función de los subprocesos implicados en la composición de textos escritos: planificación, redacción y revisión.

Estrategias de planificación: a) Analizar los elementos de la situación de comunicación (emisor, receptor, propósito, tema, etc.), b) formular con palabras el objetivo de una comunicación escrita: ¿Qué se espera conseguir?, c) consultar fuentes de información diversas: enciclopedias, diccionarios, etc., d) aplicar técnicas diversas de organización de ideas (esquemas jerárquicos, ideogramas, palabras clave, etc.), e) determinar cómo será el texto (extensión, tono, presentación, etc.), y f) elaborar borradores.

Estrategias de redacción: a) Proceder a plasmar sobre el papel las ideas; b) concentrarse selectivamente en diversos aspectos del texto; c) manejar el lenguaje para lograr el efecto deseado, y d) utilizar la sintaxis correcta y seleccionar vocabulario.

Estrategias de revisión: a) Comparar el texto producido con la planificación previa; b) leer de forma selectiva, concentrándose en distintos aspectos: contenido (ideas, estructura, etc.) o forma (gramática, puntuación, ortografía, etc.); c) dominar diversas formas de rehacer o retocar un texto: eliminar o añadir palabras o frases, utilizar sinónimos, reformulación global, y d) estudiar modelos.

Otro aporte de gran importancia lo proporcionan Heller y Thorogood (1995) quienes establecen una clasificación de estrategias metacognitivas de producción de textos o captación consciente de las capacidades y limitaciones de los procesos de pensamiento que originaron un texto escrito. Mientras mayor sea el conocimiento de lo que se sabe, del porqué de los aciertos o desaciertos, se tendrá más posibilidades de aprender, consolidar éxitos, superar deficiencias y disminuir la dependencia del azar o suerte.

Las estrategias metacognitivas sugeridas son:

Antes de escribir: a) Explicar el objetivo que se tiene cuando se escribe; b) construir ideas acerca del contenido del texto y su relación con los conocimientos previos, y c) tomar conciencia 
sobre: ¿Para quién estoy escribiendo?, ¿qué tipo de texto quiero escribir?, ¿por qué escribo?

Durante la escritura: a) Identificar las dificultades que impiden expresar las ideas; b) utilizar palabras, oraciones y párrafos para comunicarse; c) asegurar la coherencia y cohesión del texto, y d) seleccionar ideas principales y secundarias.

Después de la escritura: a) analizar la calidad del contenido: amplitud, riqueza, profundidad y precisión de las ideas; b) revisar la organización y presentación del contenido: estructura del texto y de las unidades que lo componen; c) confirmar o rechazar el estilo de la escritura: adecuación a la audiencia y a la finalidad, y d) corregir aspectos morfológicos, sintácticos, léxico, ortografía.

Otros estudios le dan valor a las interacciones sociales y las opiniones, ya que pueden permitir la creación de una escritura cooperativa con un valor funcional comunitario. De tal manera que los procesos de planificación, textualización y revisión puedan ser recíprocas entre los estudiantes.

\section{Composición de textos escritos}

Para Díaz Barriga y Hernández (2010):

La enseñanza tradicional de la composición comúnmente se ha centrado en el producto logrado (el texto final) por los estudiantes, donde el profesor señala y trata de corregir sus errores, pero donde poco o nada se enseña sobre cómo construirlos desde una perspectiva netamente retórica, discursiva y funcional; en su lugar se enseñan habilidades de "bajo nivel",... Y más aún, las prácticas de redacción son totalmente artificiales y de ejecución inocente, dado que poco se implica la composición como una actividad funcional dentro del contexto comunicativo propiamente dicho. (p. 268)

Ciertamente la actividad de composición de textos en las aulas de clase es muy poca y sin sentido, inexplicablemente el docente se ocupa de revisar caligrafía, ortografía, margen, legibilidad entre otros aspectos formales de la escritura basado en lo que los estudiantes copian, pero casi nunca se propone una actividad que despierte en ellos el deseo de escribir alguna experiencia de su vida o cambiarle el final a un cuento conocido.

Durante la composición escrita los novatos invierten menos tiempo y esfuerzo de desarrollo en comparación con los expertos. Por ejemplo, en la planificación tienden a comenzar a escribir sin 
tener claro sus objetivos y otros elementos. En la etapa de textualización, el experto desempeña un comportamiento estratégico en el cual va controlando de manera permanente lo que está escribiendo, confrontando el escrito con los objetivos iniciales y con los intereses de los posibles lectores.

Tapia (2002) define la composición escrita como:

... algo más que poner una idea por escrito. Supone intentar resolver un problema inicialmente mal definido, mediante un proceso a través del que el autor debe determinar si el producto que va logrando se ajusta a la representación que de modo anticipado se va haciendo de los requisitos que debe reunir el producto final. Se trata de un proceso estratégico que, como veremos puede enseñarse, si bien normalmente no ha sido de instrucción directa. (p. 212)

La composición escrita es una actividad fundamental que debe realizar el ser humano a lo largo de toda su vida y especialmente en el ámbito escolar, en la Educación Básica donde podrá generar nuevas ideas y expresar con más exactitud sus sentimientos y también sus opiniones.

\section{La escritura en la Educación Básica}

En el Currículo Básico Nacional (Ministerio de Educación, 1997), para el área de Lengua y Literatura en la Segunda Etapa de Educación Básica se plantea que la enseñanza de la lengua es la base indispensable de todas las áreas académicas, de los valores y un muy importante instrumento de interacción humana; además, es el fundamento de los cambios personales, sociales y culturales a los que obliga la dinámica actual.

En anteriores planteamientos se mencionó que en el área de lengua el enfoque funcional comunicativo, se ocupa del desarrollo de las competencias comunicativas entendidas como el conocimiento del sistema lingüístico y los códigos no verbales y la adecuación de su actuación lingüística a los diferentes contextos socioculturales y situacionales.

El enfoque funcional comunicativo está sustentado en las teorías del desarrollo evolutivo y el aprendizaje significativo; y los nuevos aportes lingüísticos de Vygotsky (1988), Piaget (1966, 1976) y Ausubel (1983). Dentro de esos aportes se destacan las propuestas teóricas y metodológicas de la Psicolingüística que explica los procesos de adquisición y desarrollo del lenguaje; la 
variabilidad derivada del uso de la lengua en diferentes contextos comunicativos, preocupación de la Sociolingüística y la atención a la diversidad funcional del lenguaje en la vida de los niños.

Como complemento, el Currículo Básico Nacional (Ministerio de Educación, 1997) propicia una didáctica que favorece el aprender a aprender y atiende a través de contenidos conceptuales, procedimentales y actitudinales los aprendizajes fundamentales: aprender a conocer, aprender a hacer y aprender a ser. Asimismo, estimula el acceso a la escritura y la lectura como acciones placenteras que proporcionan enriquecimiento personal. $\mathrm{Al}$ atender a los procesos de comprensión y producción tanto de la lengua oral como de la escrita, para construir así su aprendizaje.

En tal sentido, el programa de Lengua y Literatura está organizado en seis bloques de contenidos y para efectos de esta investigación se desarrolló el quinto bloque denominado, Literatura: el mundo de la imaginación.

El bloque Literatura: el mundo de la imaginación, se refiere a las experiencias de aprendizaje relacionadas con el deleite y la recreación que permite la interacción con la literatura y promueve la creatividad y el desarrollo de la fantasía en la producción espontánea de textos imaginativos. La literatura debe asumirse como el pilar fundamental para la formación de productores de textos creativos. La función imaginativa de la literatura permite al estudiante el beneficio personal, el conocimiento del acervo cultural de su contexto social, la reafirmación de su identidad y el contacto con diferentes mundos lo cual ayuda el desarrollo del pensamiento divergente (Ministerio de Educación, 1997, p. 133).

En relación con los contenidos el bloque Literatura: el mundo de la imaginación encierra muchos contenidos que un estudiante debe saber. A continuación, algunas consideraciones específicas acerca del manejo de los contenidos del bloque del área para los procesos de producción de textos escritos por ser más complejos que los de la lengua oral. El programa propone contenidos referidos a la organización de ideas, la planificación de la escritura, la estructuración coherente de acuerdo con patrones gramaticales y formales de la lengua, la revisión, la autocorrección y la reelaboración de textos.

Definitivamente, no hay que olvidar que la lengua es el instrumento que los hombres necesitan para comunicarse y, para toda persona que forma parte de una comunidad lingüística, es muy importante saber si un uso gráfico, es correcto o incorrecto, si lo que se escribe tiene coherencia e intención y se expresan los 
sentimientos al escribir. Por lo tanto, cuanto mayor y mejor sea el conocimiento de la lengua, en su manifestación escrita mayor y mejor será la comunicación con los demás.

\section{Los textos narrativos}

El texto narrativo es el más utilizado en el aula durante los primeros años de la escolaridad. Barboza et al., (2008) afirman que:

En la escuela tenemos la oportunidad de favorecer una inmersión en las historias y en las palabras de otros que nos antecedieron y dieron forma a la cultura que hoy nos encuentra. Pero ese contacto se justifica para formarnos y transformarnos y porque la narración permite apropiarse de las habilidades cognitivo lingüísticas que requiere su construcción. (p. 103)

Los textos narrativos elaborados en forma individual y colectiva son una muy buena estrategia para aplicar en el aula, ya que permitirán revelar el grado de desarrollo de las destrezas alcanzadas por los aprendices, les enseñará a ordenar sus ideas de tal forma que sus relatos se puedan comprender. También permitirán que los docentes puedan apreciar deficiencias en la expresión escrita que deberán ser atendidas de forma adecuada y oportuna.

Para Adam y Lorda (2010) el texto narrativo es una forma de expresión que cuenta hechos o historias acontecidas a sujetos, ya sea humanos (reales o personajes literarios), animales o cualquier otro ser antropomorfo, cosas u objetos; en él se presenta una concurrencia de sucesos (reales o fantásticos) y personas en un tiempo y espacio determinados.

Ambos autores coinciden en que las narraciones se pueden convertir en una alternativa para ver el mundo de una manera personal ya que ayuda a reconfigurar la propia identidad y a confrontar las historias personales con las historias de otros, en un diálogo consigo mismo.

Para Calsamiglia (2000), las narraciones son actos comunicativos que suponen la existencia de un emisor con una intención ¿Por qué narrar? y una finalidad ¿para qué narrar? Existen narraciones literarias cuya finalidad es eminentemente artística (por ejemplo, las presentes en cuentos, fábulas, leyendas, mitos y novelas, entre otros textos artísticos o de pretensiones estéticas), así como las narraciones no literarias, cuya finalidad es 
informativa (por ejemplo, noticias periodísticas, crónicas, reportajes, conversaciones en donde se relata algo, anécdotas, entre otros); en este último caso se narran hechos no ficticios.

Las narraciones se caracterizan por presentar de modo indispensable varios sucesos integrados por uno cuya ejecución es necesaria para producir el suceso siguiente; estos sucesos se realizan cronológicamente (por lo cual, en las narraciones predominan conectores o marcadores temporales, tales como "antes", "después", "al cabo de una semana", entre otros), en una serie de progresiones lineales que determinan una construcción regida por el esquema causa/efecto. El participante en una narración (a quien se denomina sujeto) es un humano, un ser, objeto, pasión, etc., humanizado (antropomórfico) o un hecho que afecta a los humanos; por lo tanto, el tema de las narraciones compete a la vida humana. En toda narración aparecerá un sujeto fijo, personaje principal o actor principal.

Los marcadores o conectores primordiales que caracterizan las narraciones son espacio-temporales y finalizadores, si bien en muchas narraciones se emplean marcadores de inicio ("Había una vez") y de complicación o transformación ("de pronto", "de repente").

Los textos narrativos frecuentemente presentan una descripción, una exposición $\mathrm{y}$, muchas veces, alguna argumentación. También dentro de una narración general (como en un cuento, una novela o una crónica) es común que se incluya una narración secundaria; para identificar a esta última, se emplean marcadores espaciotemporales que la encabezan ("un día", "en cierta ocasión", "sucedió que...", "hace muchos años..."), y de la secuencia mencionada líneas arriba.

Para comprender un texto narrativo se debe tomar en cuenta la determinación del asunto, esta es una oración donde se expone una relación breve y general de lo que ha pasado en la narración, y donde se muestra la secuencia aludida anteriormente. Otro elemento importante de todo texto narrativo es el tema, que se considerará como la idea, el sentimiento, el objeto, la actitud que sintetizaría el asunto (o en su caso la narración).

Segre (1985) lo define como "la materia elaborada en un texto". El tema se expresa en un número mínimo de palabras y se distingue del asunto porque mientras este cuenta algo de modo sucinto, el tema es la médula de lo que se habla. 
Para redactar una narración se debe tomar en cuenta que el texto narrativo no comprende únicamente textos literarios con pretensiones artísticas, es importante conocer los elementos mínimos que requiere trabajar quien desee redactar un texto narrativo. Según Adam y Lorda (2010), estos elementos son los siguientes: tema, sujeto fijo, lugar y tiempo, asunto, relaciones actanciales, público, argumento, entre otros.

\section{MÉTODO}

L a propuesta metodológica abordada en este estudio estuvo sustentada en el enfoque cualitativo para estudiar la realidad desde una perspectiva interpretativa.

Para Barrantes (2001), la investigación cualitativa refiere una concepción inductiva, basada en el proceso y que tiende a la búsqueda de teorías pero no de leyes exactas. Por ello se realizaron observaciones, con la finalidad de poder dar respuesta a los objetivos de la presente investigación y de esta forma poder proponer un plan de acción.

En cuanto al diseño empleado fue una investigación de campo, que para Arias (2006) consiste en "la recolección de datos directamente de los sujetos investigados, o de la realidad donde ocurre los hechos (datos primarios), sin manipular o controlar variable alguna, es decir, el investigador obtiene la información pero no altera las condiciones existentes" (p. 31). De allí que se fue al propio escenario con las personas involucradas desde el mismo contexto, y de forma natural se creó un clima de confianza y aceptación por parte de los sujetos de estudio.

El nivel empleado para el tratamiento de los datos fueron los niveles descriptivo e interpretativo.

Finalmente, el carácter descriptivo e interpretativo permitió que se recolectaran los datos de la manera más meticulosa posible y así aportar el insumo necesario al análisis de las estrategias utilizadas por los docentes, en el proceso de composición de textos narrativos en cuarto grado de Educación Primaria en el Grupo Escolar Guatire.

El escenario de la investigación fue una institución ubicada en el sector las Barrancas, Calle los Lirios, Carretera Nacional Guarenas-Guatire (Venezuela), con el nombre de Grupo Escolar Guatire.

Los sujetos de la presente investigación fueron tres (3) docentes de cuarto grado que laboran en la institución, con una 
matrícula de veinticuatro (24) estudiantes por aula con edades comprendidas entre nueve (9) y once (11) años de edad. La selección de estos fue de manera intencionada, tomando en cuenta la sección que presentó más debilidad en la composición de textos narrativos.

Las técnicas empleadas fueron la entrevista semiestructurada y la observación participante. Estas técnicas se apoyaron en los instrumentos con los que se recolectó la información los cuales fueron: el guion de entrevista y una guía de observación.

Las entrevistas en esta investigación se llevaron a cabo en momentos libres de los docentes, en fechas previamente acordadas y en sus respectivas aulas de clase.

Las observaciones fueron pautadas con anterioridad y se realizaron a través de la asistencia a tres salones de clases en tres jornadas diarias; con la intención de observar e identificar las estrategias instruccionales utilizadas por los docentes para promover la composición de textos narrativos en estudiantes de cuarto grado.

Por medio del instrumento registro descriptivo se sistematizaron las observaciones diarias basadas en la planificación.

El guion de entrevista también se utilizó como instrumento. Este quedó estructurado con diez preguntas orientadoras que sirvieron para indagar sobre las estrategias que emplean los docentes a fin de dirigir el proceso de composición de textos narrativos en cuarto grado; además se aplicó una guía de observación con 13 aspectos a observar durante la asistencia a las clases, en el área de lengua en este nivel.

\section{Procedimiento de la investigación}

Etapa I. Estrategias empleadas por los docentes. En esta fase se realizó una revisión de la literatura relacionada con el tema que sirvió de sustento a la investigación. Se delimitó la descripción de la problemática elegida para describir las debilidades que presentaron los docentes al orientar la composición de textos narrativos y los estudiantes al componer textos narrativos.

Paralelamente, se diseñaron dos instrumentos: un guion de entrevista semiestructurada y una guía de observación participante los cuales fueron utilizados para recoger la información. Dichos instrumentos fueron validados por tres 
expertos: uno en diseño de instrumentos de evaluación y dos en estrategias de aprendizaje, uno de ellos también especialista en Lengua y Literatura, todos docentes activos del Instituto Pedagógico de Miranda José Manuel Siso Martínez. Seguidamente, se acordaron las citas con los sujetos participantes y se realizaron las tres entrevistas; es decir, una a cada uno de los docentes de cuarto grado (A, B y C). Luego, se realizaron tres observaciones identificando como informantes (A, B y C), previa cita a los docentes participantes al término de las entrevistas y con permiso de la dirección de la institución.

Etapa II. Diseño de las estrategias instruccionales. El diseño de las estrategias se llevó a cabo en tres fases:

Fase I. Revisión de material bibliográfico relacionado con la composición de textos narrativos para contextualizar la situación problemática y describirla. Se elaboró el marco referencial y la metodología, donde la investigadora después de aplicar cada una de las técnicas y obtener la información necesaria realizó el análisis de las estrategias instruccionales utilizadas por los docentes dirigidas a la composición de textos narrativos.

Fase II. Se procedió a diseñar las estrategias instruccionales basadas en la enseñanza estratégica para abordar la composición de textos narrativos en estudiantes de cuarto grado de Educación Primaria del Grupo Escolar Guatire, estado Miranda.

\section{RESULTADOS}

Etapa I. Estrategias empleadas por los docentes. Luego del análisis e interpretación de los datos obtenidos durante las entrevistas y las observaciones realizadas, se establecieron las categorías apriorísticas y las subcategorías las cuales se presentan en el Cuadro 1.

Cuadro 1. Categorías y subcategorías

\begin{tabular}{ll}
\hline \multicolumn{1}{c}{ Categorías } & \multicolumn{1}{c}{ Subcategorías } \\
\hline \multirow{2}{*}{ Estrategias } & 1.- Activación de la atención \\
Preinstruccionales & 2.- Presentación del objetivo \\
& 3.- Activación de los conocimientos previos \\
& \\
& 1.- Presentación de la nueva información \\
Estrategias & 2.- Focalización de la atención (uso de diversos \\
Coinstruccionales & semánticas como presentaciones, láminas, redes \\
& 3.- Práctica del contenido
\end{tabular}




\begin{tabular}{ll}
\hline \multicolumn{1}{c}{ Categorías } & \multicolumn{1}{c}{ Subcategorías } \\
\hline Estrategias & 1.- Resume la lección \\
Posinstruccionales & 2.- Promoción de la transferencia de lo aprendido \\
& \\
& 1.- Medios y recursos empleados \\
Componentes del & $\begin{array}{l}\text { 2.- Técnicas utilizadas } \\
\text { proceso instruccional }\end{array}$ \\
& 3.- Organización del grupo \\
& 4.- Evaluación aplicada \\
\hline
\end{tabular}

Etapa II: Diseño de las estrategias instruccionales. Sobre la base de los resultados obtenidos en la etapa I, la cual consistió en determinar las estrategias instruccionales utilizadas por los docentes dirigidas a la composición de textos narrativos en cuarto grado de Educación Primaria del Grupo Escolar Guatire, y considerando las bases teóricas y conceptuales que sustentan este estudio, se diseñaron cinco estrategias instruccionales, las cuales tienen como finalidad promover la enseñanza estratégica para abordar la composición de textos narrativos; además de proporcionar herramientas a los docentes para mejorar su práctica pedagógica y actualizar su formación profesional.

Después de diseñadas las estrategias, se elaboró un material instruccional, titulado Componiendo cuentos y fábulas (Pérez, 2018), cuya presentación contiene una atractiva diagramación y su estructura se organizó de la siguiente manera: introducción; estructura del material instruccional; fundamentación teórica: enfoque cognitivo, teoría psicogenética de Jean Piaget, enfoque sociocultural de Vygotsky, enseñanza estratégica; las estrategias instruccionales: nombre de la estrategia, organización de la secuencia, objetivo, contenido, momentos, eventos, medios y recursos, técnicas y organización; una reflexión y las referencias.

En el Cuadro 2 se evidencia información resumen de cada una de las cinco estrategias. 
Cuadro 2. Nombre de la estrategia instruccional, objetivo y contenido

\begin{tabular}{|c|c|c|}
\hline $\begin{array}{l}\text { Nombre de la } \\
\text { estrategia }\end{array}$ & Objetivo & Contenido \\
\hline $\begin{array}{l}\text { Estrategia \# } 1 \\
\text { Aprendiendo a crear } \\
\text { párrafos }\end{array}$ & $\begin{array}{l}\text { Construir oraciones } \\
\text { para crear párrafos. }\end{array}$ & $\begin{array}{l}\text { Elementos que integran } \\
\text { el texto: la oración y el } \\
\text { párrafo }\end{array}$ \\
\hline $\begin{array}{l}\text { Estrategia \#2 } \\
\text { Cambiando letras } \\
\text { encontramos palabras } \\
\text { nuevas }\end{array}$ & $\begin{array}{l}\text { Reconocer las clases } \\
\text { de palabras y la } \\
\text { función que cumplen } \\
\text { dentro de la oración. }\end{array}$ & $\begin{array}{l}\text { Sustantivos, pronombres, } \\
\text { adjetivos, verbos, } \\
\text { adverbios, artículos y } \\
\text { preposiciones }\end{array}$ \\
\hline $\begin{array}{l}\text { Estrategia \#3 } \\
\text { Conociendo las etapas } \\
\text { para redactar un texto } \\
\text { narrativo }\end{array}$ & $\begin{array}{l}\text { Aprender a planificar, } \\
\text { a redactar y a corregir } \\
\text { un texto narrativo. }\end{array}$ & $\begin{array}{l}\text { Textos narrativos: el } \\
\text { cuento y la fábula }\end{array}$ \\
\hline $\begin{array}{l}\text { Estrategia \# } \mathbf{4} \\
\text { Componiendo una fábula } \\
\text { a ocho manos }\end{array}$ & Componer una fábula. & $\begin{array}{l}\text { Textos narrativos: el } \\
\text { cuento y la fábula }\end{array}$ \\
\hline $\begin{array}{l}\text { Estrategia \#5 } \\
\text { Componiendo un cuento } \\
\text { a cuatro manos }\end{array}$ & Componer un cuento. & $\begin{array}{l}\text { Textos narrativos: el } \\
\text { cuento y la fábula }\end{array}$ \\
\hline
\end{tabular}

\section{CONCLUSIONES}

os docentes en este nivel de Educación Primaria entrevistados y observados no emplean estrategias instruccionales novedosas ni creativas conformes a orientar a los aprendices en la composición de textos narrativos; más concretamente, solo se limitan a cubrir los contenidos curriculares sin planificar actividades que permitan desarrollar en los estudiantes habilidades para aprender de forma estratégica el 
proceso de composición escrita. Las actividades llevadas a cabo dentro del aula están más orientadas a copiar que a componer, sin motivar a los estudiantes a transferir el conocimiento o a ponerlo en práctica en la vida cotidiana.

Se diseñaron cinco estrategias instruccionales basadas en la enseñanza estratégica, atendiendo a los intereses y motivaciones de los estudiantes y por ende permitirán promover la composición de textos narrativos en estudiantes de cuarto grado de Educación Básica, ya que se conectará a los estudiantes con sus experiencias previas y así conocer lo que saben, que les gusta y qué necesitan para aprender.

De igual manera, unas estrategias instruccionales bien diseñadas bajo el enfoque de la enseñanza estratégica tendrán efectos positivos, por cuanto se logrará en los educandos actitudes más reflexivas orientadas a tomar conciencia y decisiones al momento de realizar un texto escrito; aprenderán lo significativo de expresarse a través de la escritura y a desarrollar el proceso de composición escrita.

Finalmente, se puede señalar que el principal rol del docente en los procesos de enseñanza y aprendizaje consiste en planificar secuencias didácticas que ayuden, impulsen y motiven a los aprendices a escribir textos narrativos de forma placentera y espontánea. Para ello, los docentes deben realizar estudios de actualización a fin de ser innovadores y creativos en la planificación de estrategias adaptadas a los intereses de cada grupo de estudiantes.

\section{REFERENCIAS}

Adam, M. y Lorda, C. (2010). Lingüística de los textos narrativos. Barcelona. España: Ariel

Alfonzo, A. (2003). Estrategia Instruccional. Recuperado de www.medusa.unimet.edu.ve/educación/fbi21/estrategias.pdf

Arias, F. (2006). El Proyecto de investigación. Introducción a la metodología científica. (5 $5^{\mathrm{a}}$ ed.). Caracas: Episteme

Ausubel, D. (1983). Psicología de la Educación. México DF: Trillas

Barboza, F. Aquirre, R. y Peña, .J. (2008). Una experiencia para estimular la composición escrita en estudiantes universitarios de formación docente. Revista Saber-ULA. Educere. 12 (40)

Barrantes, R. (2001). Investigación: Un camino al conocimiento, un enfoque cualitativo y cuantitativo. San José, CR.: EUNED

Caldera, R. (2003). El enfoque cognitivo de la escritura y sus consecuencias metodolóqicas en la escuela. Educere. 6 (20)

Calsamiglia, H. (2000). Estructura y funciones de la narración. Textos 25. Barcelona: Graó 
Cassany, D. (1990). Enfoque didáctico para la Enseñanza de la Expresión Escrita: La comunicación de Lenguaje y Educación. Madrid: Aprendizaje

Cassany, D., Luna, M. y Sanz, G. (2000). Enseñar lengua. Barcelona, España: Graó

Díaz Barriga, F. y Hernández, G. (2010). Estrategias docentes para un aprendizaje significativo. Una interpretación Constructivista. México, DF: Mc Graw Hill. pp. 268, 269

Díaz L. (2005). Redacción de textos expositivos: una propuesta pedagógica. Educere, 9(30), 301-309

Feo, R. (2010). Estrategias instruccionales para promover el aprendizaje estratégico en estudiantes del Instituto Pedagógico de Miranda José Manuel Siso Martínez. En Revista Universitaria de Investigación Sapiens, 11 (2). Caracas. Recuperado

de http://www2.scielo.org.ve/scielo.php?script=sci arttext\&pid=S 1317-58152010000200007\&lng=es\&nrm=iso

Gagné, L. y Briggs, M. (1978). Planificación de la enseñanza. Sus principios. México: Trillas

Gaskins, J. y Pressley, L. (2006). La estrategia instruccional. Recuperado de http://giddet.psicol.unam.mx/giddet/biblioteca/basica/docente estrategico monereo.pdf

Heller, M. y Thorogood, L. (1995). Hacia un proceso de lectoescritura reflexivo y creativo. Caracas: Educativa

Lomas, C. (2001). El aprendizaje de la comunicación en las aulas. Buenos Aires: Paidós

Ministerio de Educación (1997). Currículo Básico Nacional, Programa de Educación Básica. II Etapa

Monereo, C. (2004). La enseñanza estratégica: Enseñar para la autonomía. Barcelona, España: Graó

Pérez B., N. (2018). Estrategias instruccionales basadas en la enseñanza estratégica para abordar la composición de textos narrativos en cuarto grado de Educación Primaria del Grupo Escolar Guatire-Estado Miranda (trabajo de maestría). Universidad Pedagógica Experimental Libertador (UPEL), Miranda, Venezuela

Piaget, J. (1966). Teoría Genética del Aprendizaje. Buenos Aires: Psique

Piaget, J. (1976). Psicología y pedagogía. Barcelona, España: Ariel

Segre, C. (1985). Principios de análisis del texto literario. Caracas: Crítica

Tapia, J. (2002). Motivación y aprendizaje en el aula. Madrid: Santillana

Vygotsky, L. (1988). El desarrollo de los procesos psicológicos superiores. México: Editorial Crítica, Grupo editorial Grijalbo

Vygotsky, L. (1979). El desarrollo de los procesos psicológicos superiores. Barcelona, España: Visor 